\title{
A comparative histopathological study of argon and krypton laser irradiations of the human retina
}

\author{
JOHN MARSHALL AND ALAN C. BIRD \\ From the Department of Visual Science and Clinical Ophthalmology, Institute of Ophthalmology, \\ Moorfields Eye Hospital, Judd Street, London WC1H $9 Q S$
}

SUMMARY A series of comparative exposures to both argon and krypton lasers have been made at 3 locations in a human retina - the fovea, the macula, and intraretinal vessels. In the fovea argon irradiations resulted in damage to both the inner and outer retinal layers as a result of absorption within the pigment epithelium and the macular pigment, while krypton exposures damaged the outer retina and the choroid. In the macula both systems resulted in damage to the outer retina, and again sufficient krypton radiation passed into the choroid to induce blood vessel occlusion, haemorrhage, and oedema. When intraretinal vessels were irradiated, only with argon was sufficient energy absorbed within the vessels to damage them or their surroundings in the inner retina. The implications of these findings are discussed in relation to the therapeutic uses of lasers.

Both the initial destruction and the secondary tissue responses induced by the coagulative action of laser radiation on retinal tissue are exploited therapeutically in ophthalmology. The primary reaction in most clinical exposures is one of tissue destruction resulting from the forces generated by the thermal degradation of the incident energy absorbed in the target tissue (Marshall, 1970). The amount of energy absorbed is a function of the wavelength of the incident radiation and the absorption characteristics of the irradiated media. The degree and extent of destruction within a tissue are also related to the magnitude of the exposure energy, the area of irradiation, and the duration of the exposure. Throughout the visible spectrum the predominant absorption site in the retina is the pigment epithelium, and the physical parameters of most clinical exposures are such that only this layer and the overlying photoreceptor cells are damaged. In the 1960 s such a burn configuration with damage confined to the outer retina was not considered useful for the treatment of many neovascular conditions, especially those in which forward new vessels occurred. At that time the ideal system was considered to be one with which vessels could be directly irradiated by a source with a wavelength which would be absorbed by haemoglobin. The argon laser met such requirements (L'Esperance, 1968), and for some time various protocols for

Correspondence to Professor Bird. direct irradiation of neovascular systems were developed (Little et al., 1970). Such techniques continue to be used in the treatment of subretinal neovascular complexes or disciform lesions (Gass, 1971, 1973; Bird, 1974).

Direct treatment of new blood vessels in diabetes (Little, 1973) has been largely replaced by peripheral ablation, whereby large areas of the outer retinal layers in the peripheral fundus are destroyed (L'Esperance, 1975a). Furthermore, in disciform lesions at least part of the new vessels are close to the pigment epithelium, and therefore, irradiation energy absorbed within this layer may be responsible for blood vessel closure. Thus, at present, treatment regimens for both these neovascular lesions are utilising the pigment epithelium as the absorption site and are not dependent upon the blue-green $(488,514.5 \mathrm{~nm})$ emission of the argon laser. In some circumstances there is evidence that the use of these wavelengths may be counterproductive, resulting in undesirable complications. For example, when treating disciform lesions close to the fovea approximately $70 \%$ of the incident energy at $488 \mathrm{~nm}$ will be absorbed by macular pigment and may result in nontherapeutic destruction in the inner retinal layers and prevent irradiation of the target tissue (Marshall et al., 1974, 1975). Further, when argon irradiation falls upon intraretinal vascular elements, unnecessary damage may be induced in adjacent neurones (McLeod et al., 1977). For these reasons it may be desirable to use lasers that emit 
red light. Historically such lasers have not been favourably received by ophthalmologists, but the problems with these early lasers have now been shown to be unrelated to wavelength and to have resulted from instrument design. The poor results obtained with the ruby lasers $(694.3 \mathrm{~nm})$ may have been due to short pulse duration of these devices, and the limited experiments with helium neon $(632.8 \mathrm{~nm})$ failed mainly because of inefficient optics in the delivery systems (Manson et al., 1972). There are now commercial systems available that enable either argon $(488 \mathrm{~nm})$ or krypton $(641 \mathrm{~nm})$ radiation to be used for the treatment of retinal disease. Thus for the first time the relative merits of red and green light may be assessed by using continuous wave lasers with similar optical systems.

The present paper describes the histopathology of both argon and krypton irradiations in a variety of locations on the fundus and relates their morphology to the physical parameters of the irradiating sources.

\section{Methods and materials}

Two different laser systems were used in the present study, a Coherent Radiation 800 argon laser, and a Lasertek krypton laser. Although the Lasertek system may be used to deliver either krypton or argon radiation we thought that the use of the Coherent Radiation argon system would allow a more useful comparison as this model has been widely adopted in clinical centres. Both of these lasers have integral power monitors and all figures quoted are those registered on the manufacturers' instruments. No study was undertaken of the relative energy distribution within the laser beams.

Exposures were delivered via the integral slitlamp systems in conjunction with a Goldmann fundus contact lens. One eye of a single patient was exposed 20 hours prior to enucleation for a malignant melanoma of the anterior choroid; the retinal detachment in this patient did not involve the posterior pole.

In a peripheral area of retina trial exposures were undertaken in order to adjust power levels so that approximately equal tissue responses were achieved. These exposures showed that for any given image size and exposure time krypton exposures always required higher power levels to achieve an equiretinal response to that of argon.

Two groups of $0 \cdot 2$ to 0.5 second exposures were made with each type of laser. In the macula a series of 8 argon exposures were made in a line on the superonasal to inferotemporal axis and which traversed the fovea $(50 \mu \mathrm{m}$ image size, $87 \mathrm{~mW})$. A similar number of krypton exposures were made at similar distances from the fovea at right-angles to lines of argon burns $(50 \mu \mathrm{m}$ image size, $200 \mathrm{~mW})$. One $150 \mathrm{~mW}$ krypton exposure was made at the foveola.

The second group of exposures involved the irradiation of similar calibre arterial and venous elements of the intraretinal circulation with both argon $(200 \mu \mathrm{m}, 300 \mathrm{~mW})$ and krypton $(200 \mu \mathrm{m}$, $600 \mathrm{~mW}$ ) systems.

The eye was enucleated and processed for both light and electron microscopy as previously described (Marshall et al., 1975).

\section{Results}

Ophthalmoscopic observations on the central group of argon lesions confirmed earlier findings that within $2^{\circ}$ of the fovea the morphology of the lesions changed with a coagulation site becoming apparent in the inner retina, and that the intensity of such coagulations increased as the distance from the fovea decreased. No such inner retinal damage was observed in any of the krypton exposures (Fig. 1).

Differences were also observed between the two types of irradiation on the vascular elements. Both krypton and argon induced lesions were seen as pale white areas of coagulation at the level of the pigment epithelium. With krypton lesions the vessel and inner retina appeared unaffected clinically (Fig. 2) whereas with argon the vessel lum?n was narrowed, there was haemorrhages, and opacification of the inner retina (Fig. 2). At 20 hours these

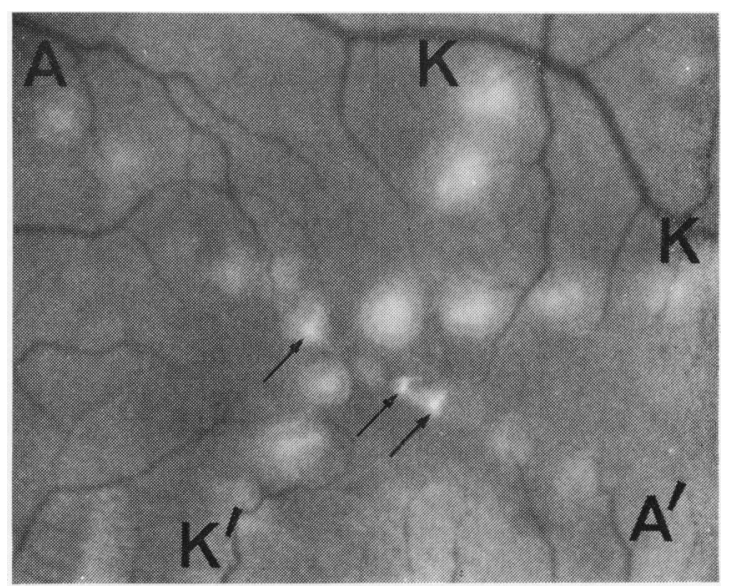

Fig. 1 Fundus photographs of the macular region immediately after irradiation by both argon $\left(A A^{\prime}\right)$ and krypton $\left(K K^{\prime}\right)$ lasers. Damage within the inner retinal layers can be seen in the 3 argon lesions nearest to the fovea (arrowed). Such damage was not produced by any of the krypton exposures 
Fig. 2 Fundus photograph of major retinal vessels immediately after irradiation by either argon (A) or krypton $(K)$ lasers. The vessels irradiated with the argon laser characteristically show a narrowed lumen, while those exposed to krypton radiation show little effect

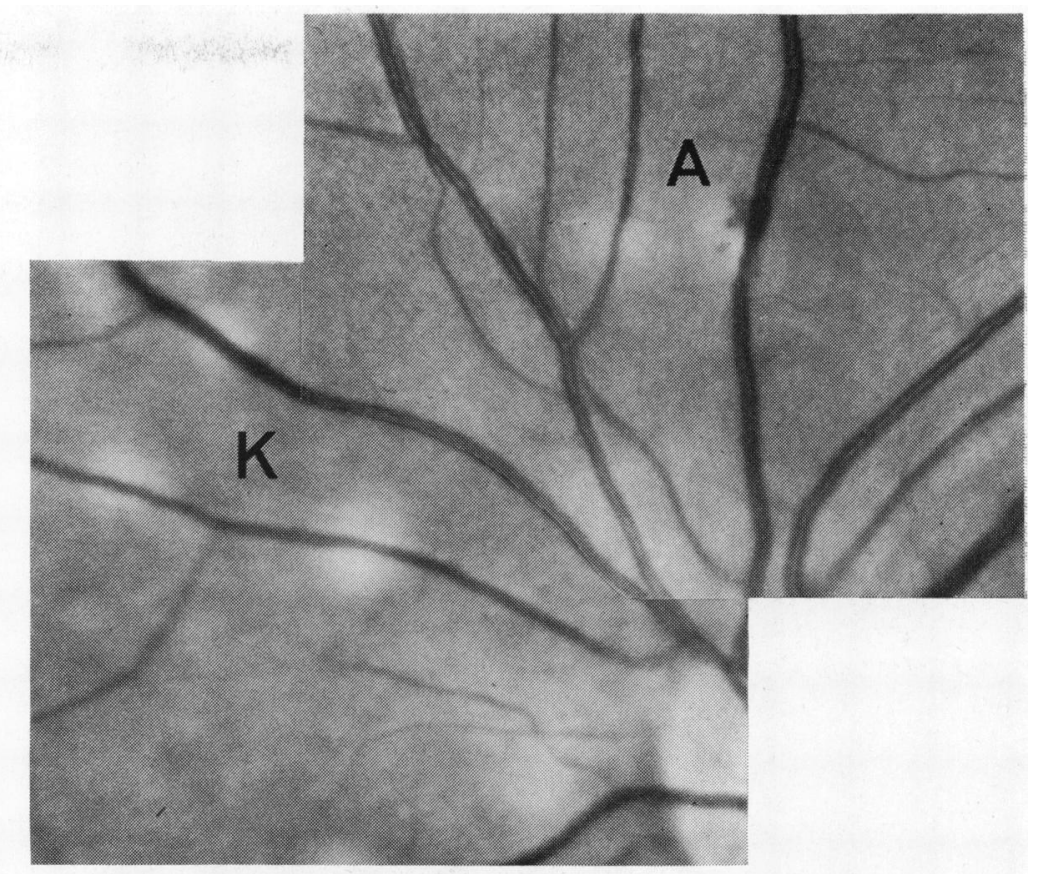

argon lesions acquired an increased opacification consisting of small fluffy white crescent-shaped changes in the inner retinal layers on the side of the lesion adjacent to the optic disc.

RETINAL DAMAGE

Irradiations of the macula

All irradiations resulted in damage to the pigment epithelium and overlying photoreceptor cells throughout the area of exposure (Fig. 3).

At distances greater than $2^{\circ}$ from the fovea argon and krypton lesions had similar morphology, except that pigment epithelial changes appeared more severe in the former. Thus in both cases the cells were either shrunken or swollen, but more extracellular space was apparent in argon induced lesions, especially in the region between the base of the cells and Bruch's membrane. In the periphery of both types of lesion the changes between irradiated and nonirradiated epithelial cells were quite marked, and cell movement had resulted in an annular zone of denuded Bruch's membrane (Fig. 3b). Some epithelial cells adjacent to irradiated areas had budded off Bruch's membrane and could be seen together with macrophages in the subretinal space between the outer segments of the photoreceptor cells (Fig. 3b, c).

The electron microscopy of subcellular changes within argon irradiated pigment epithelial cells has been previously described (Marshall et al., 1975), and in the present study a similar spectrum of damage was seen in cells irradiated by krypton.

Damage to the neural retina in this region was confined to the photoreceptor cells. Both argon and krypton irradiations resulted in areas of disorganised outer and inner segments of photoreceptor cells, which were always associated with, but smaller than, the areas of pigment epithelial disturbance. Receptor nuclei in the irradiated areas were pyknotic, though in all lesions some nuclei appeared normal. Degenerative changes were also seen in the inner connecting fibres of damaged photoreceptor cells, which were abnormally densely stained where they traversed the fibre layer of Henle. No laser induced changes were observed in the inner retinal layers of any lesions in this region of retina.

\section{Irradiations of the fovea}

Irradiation within $2^{\circ}$ of the fovea showed marked differences in damage distribution in the neural retina which were related to the irradiating wavelength (Fig. 4a).

In argon lesions in this area damage to the outer retinal layers was less severe than those seen in the outer macula. The pigment epithelial cells still showed displacement of their cell boundaries, but in equienergetic exposures the volume changes and vacuolation were less than in the more peripheral lesions. In the photoreceptor cell layer changes similar to those previously described were observed, 


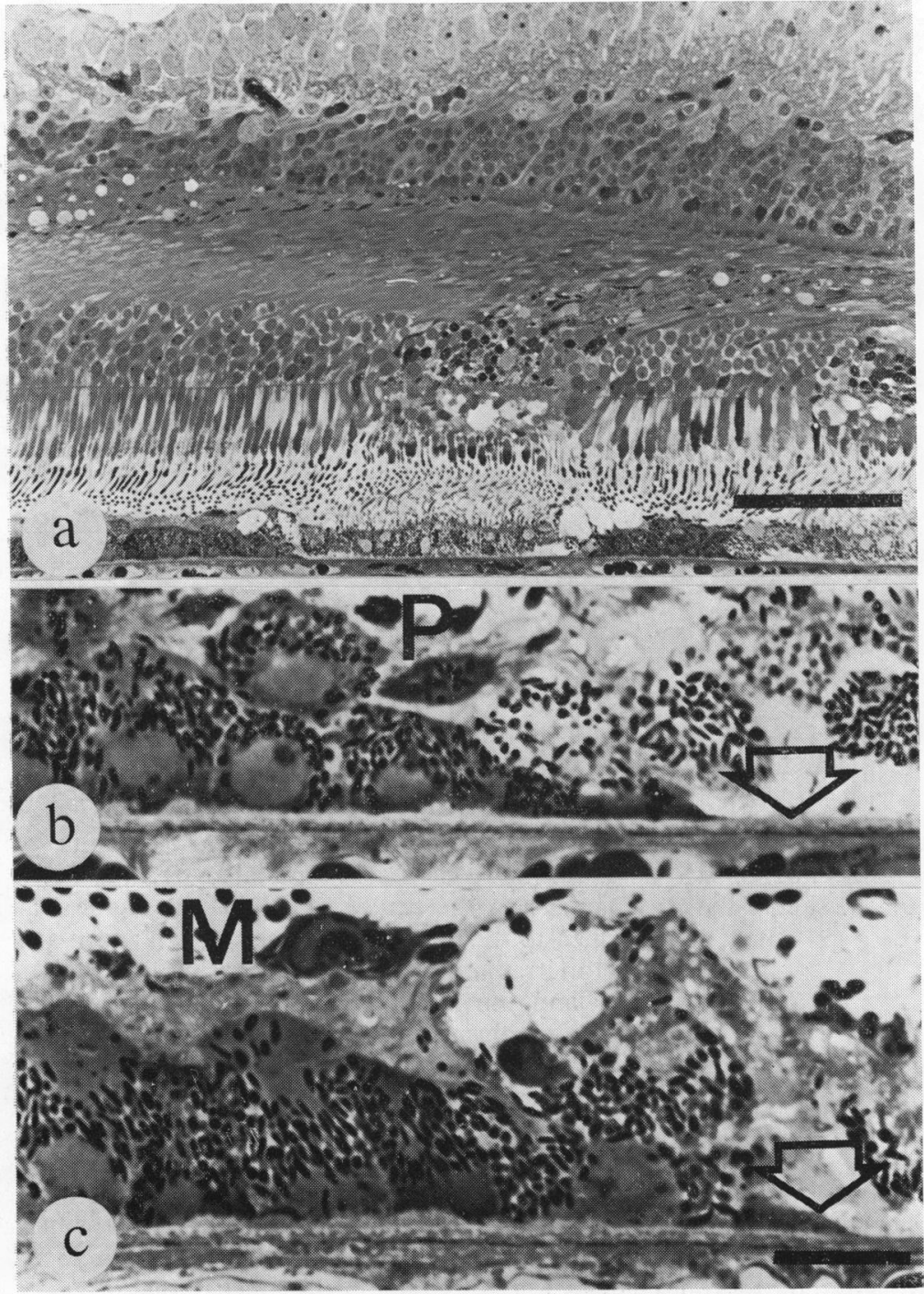

Fig. 3 (a) Light micrograph of a krypton laser induced lesion in the macula, 20 hours after exposure. Damage is confined to the pigment epithelium and the overlying photoreceptor cells. The marker is $100 \mu \mathrm{m}$. (b) (c) Light micrographs of human pigment epithelium adjacent to krypton laser lesions (arrowed) in the macula. In (b) pigment epithelial cells $(P)$ can be seen budding off Bruch's membrane, while in (c) a macrophage (M) can be seen in the subretinal space. The marker is $10 \mathrm{um}$

but again fewer cells were involved (Fig. 4b). All argon exposures resulted in gross vacuolation and tissue displacement in the inner retinal layers (Fig. 4). This second damage locus was centred on the inner nuclear layer but involved both of the plexiform layers and the ganglion cells. In all cases serial sections demonstrated that, while the inner and outer retinal damage sites were coaxial, they were discrete.

Krypton induced lesions in this region of the retina were identical to those produced in the outer macula, in that damage was confined to the outer retina, with the exception of a slightly more marked response within the pigment epithelium (Fig. 4).
Thus in the fovea argon and krypton induced changes in this layer appeared quite similar.

\section{Irradiations of intraretinal blood vessels}

The larger image sizes and higher power densities used in this part of the study resulted in larger areas of damage with more severe cellular changes in the outer retina.

In the argon induced lesions changes were observed both in the irradiated vessels, and in the adjacent nerve fibres (Fig. 5). Both arterial and venous vessels showed damage or displaced endothelial cells, and a high incidence of white blood cells within the irradiated areas. Immediately 
Fig. 4 (a) Light micrograph of both an argon $(A)$, and a krypton $(K)$ induced laser lesion in the fovea. While both lesions show damage to the retinal pigment epithelium and overlying photoreceptor cells, only the argon induced lesion shows a discrete area of damage within the inner retina. The marker is $100 \mathrm{\mu m}$. (b) Electron micrograph of the damaged outer segments within an area of argon irradiated retina at the fovea. Within 20 hours of exposure macrophages (arrowed) can be seen engulfing these elements of damaged cells. The marker is $4 \mu \mathrm{m}$.

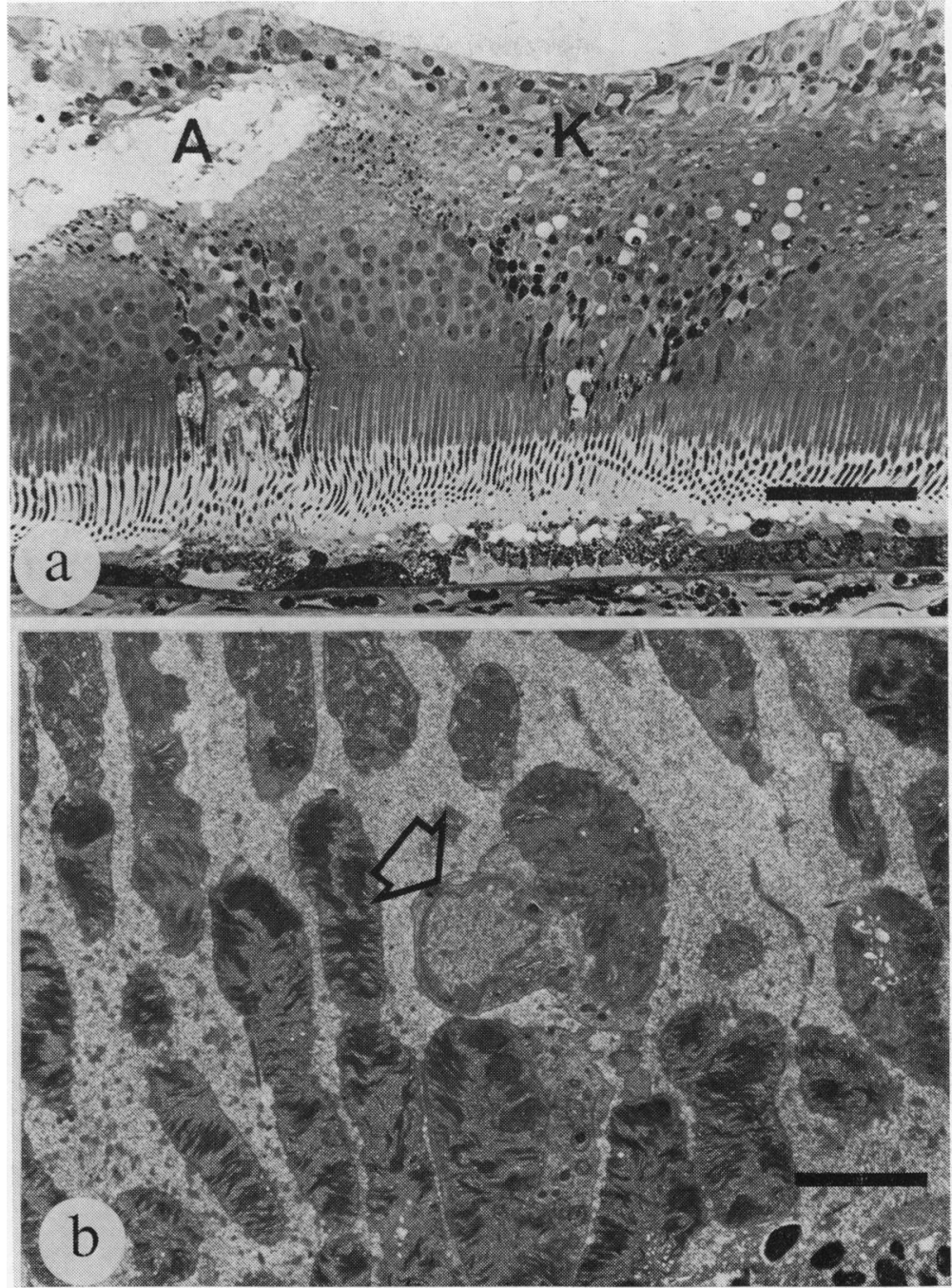

adjacent to the basement membranes of some vessels small annular areas of vacuolation were observed, and in other lesions small haemorrhages occurred into the inner plexiform layer (Fig. 5). The most striking changes seen in association with areas of argon irradiated vessels were large spheroidal axonal swellings in the nerve fibre layer. The cross-section diameters of axons in this region of the retina were normally in the range of between 0.5 and $4 \mu \mathrm{m}$; however, those of the swollen portions were between $8 \mu \mathrm{m}$ and $16 \mu \mathrm{m}$. These swellings, sometimes designated 'cytoid bodies', were found on both sides of blood vessels that ran parallel with the ganglion cell axons, but only on the side adjacent to the disc where vessels crossed the ganglion cell axons at right angles (Figs. 5, 6). On electron micro- scopy these axonal swellings were seen to contain large numbers of degenerate mitochondria, neurofilaments, and lysosomes, so densely packed that the normal axoplasm was almost excluded (Fig. 6). There were also membranous whorls and lipid bodies indicating membrane breakdown. Where vessels that ran at right-angles to the ganglion cell axons had been irradiated, less swollen but abnormally enlarged axons were also observed on the side of the vessel remote from the optic disc by electron microscopy. These swollen regions contained few organelles but had abnormal concentration of neurofilaments lining the axolemma. In all lesions some axons within the irradiated area appeared normal.

Increase in the staining density of some compo- 


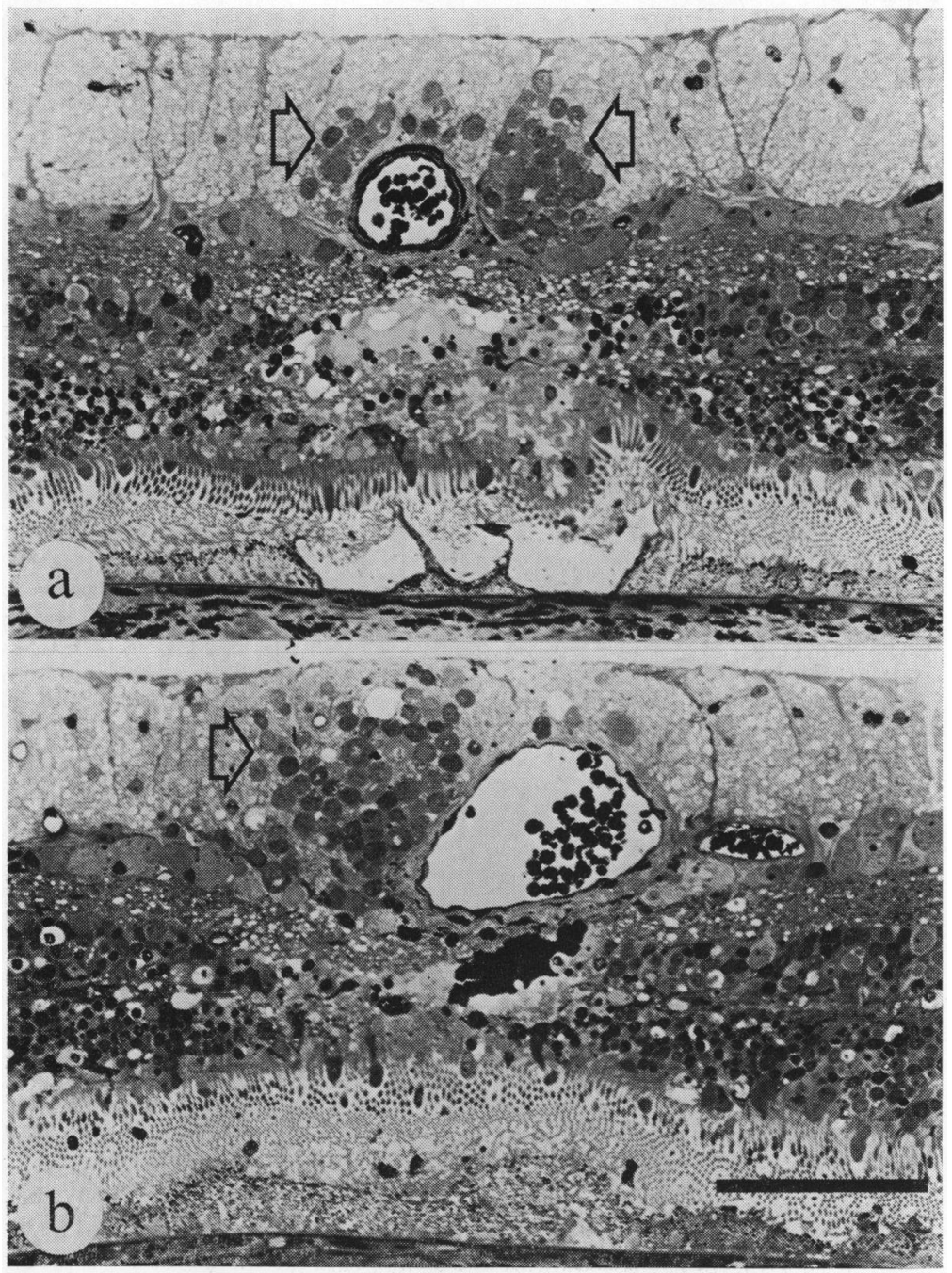

Fig. 5 Light micrographs of (a) an artery and (b) a vein 20 hours after irradiation by an argon laser. (a) The nerve fibres in this region ran obliquely over the artery on their passage to the disc and hence distended nerve fibres (arrowed) can be seen surrounding the vessel. This lesion also shows a central region of less damaged pigment epithelium lying under the artery resulting from the attenuation of incident energy by absorption within the vessel. (b) In this lesion the nerve fibres ran more at rightangles to the vein and the hence distended axons (arrowed) are predominantly on the side nearest to the disc. This exposure resulted in a small haemorrhage into the inner nuclear layer. The marker is $100 \mathrm{\mu m}$

nents of the Müller's fibres were seen, but this was largely unrelated to damage associated with intraretinal vasculature.

Damage to the neural retina induced by krypton irradiation of blood vessels was almost exclusively confined to photoreceptor cells, the exceptions being occasional densely stained Müller's fibre elements in the inner retinal layers (Fig. 7a). No damage was observed in any of the irradiated vessels, and no changes could be found in the adjacent axons of ganglion cells (Fig. 7b).

\section{CHOROIDAL DAMAGE}

In both argon and krypton irradiations there were changes in the underlying choriocapillaris adjacent to damaged pigment epithelial cells. In most lesions the capillaries were occluded throughout the irradiated areas. Blood stasis had resulted in the lumen of the vessels being either packed with degenerate blood cells or collapsed and containing remnants of endothelial cells and fibrin. Associated with these vascular changes were areas of oedema in the inner layers of the choroid. In most argon lesions damage was limited to the choriocapillaris.

By contrast vascular damage deep in the choroid was a characteristic of krypton lesion. These vascular lesions were always associated with morphological changes in adjacent choroidal melanocytes. In man these cells are usually relatively uniformly pigmented, with many branch-like processes extending from their cell bodies (Fig. 8a, b, c). Beneath areas of krypton induced damage to the retina they had lost 
Fig. 6 (a) Light micrograph of distended axons adjacent to an irradiated retinal vein. The pseudonuclei (arrowed) resulted in the term 'cytoid bodies' being given to these systems. The marker is $20 \mu \mathrm{m}$. (b) Electron micrograph of one such swollen axon showing that the pseudonuclei are a region filled with neurotubules, while the periphery contains many shrunken and degenerate mitochondria. Axons of normal dimensions (arrowed) can be seen adjacent to those showing damage, and $(V)$ is the vein. The marker is $2 \mu \mathrm{m}$

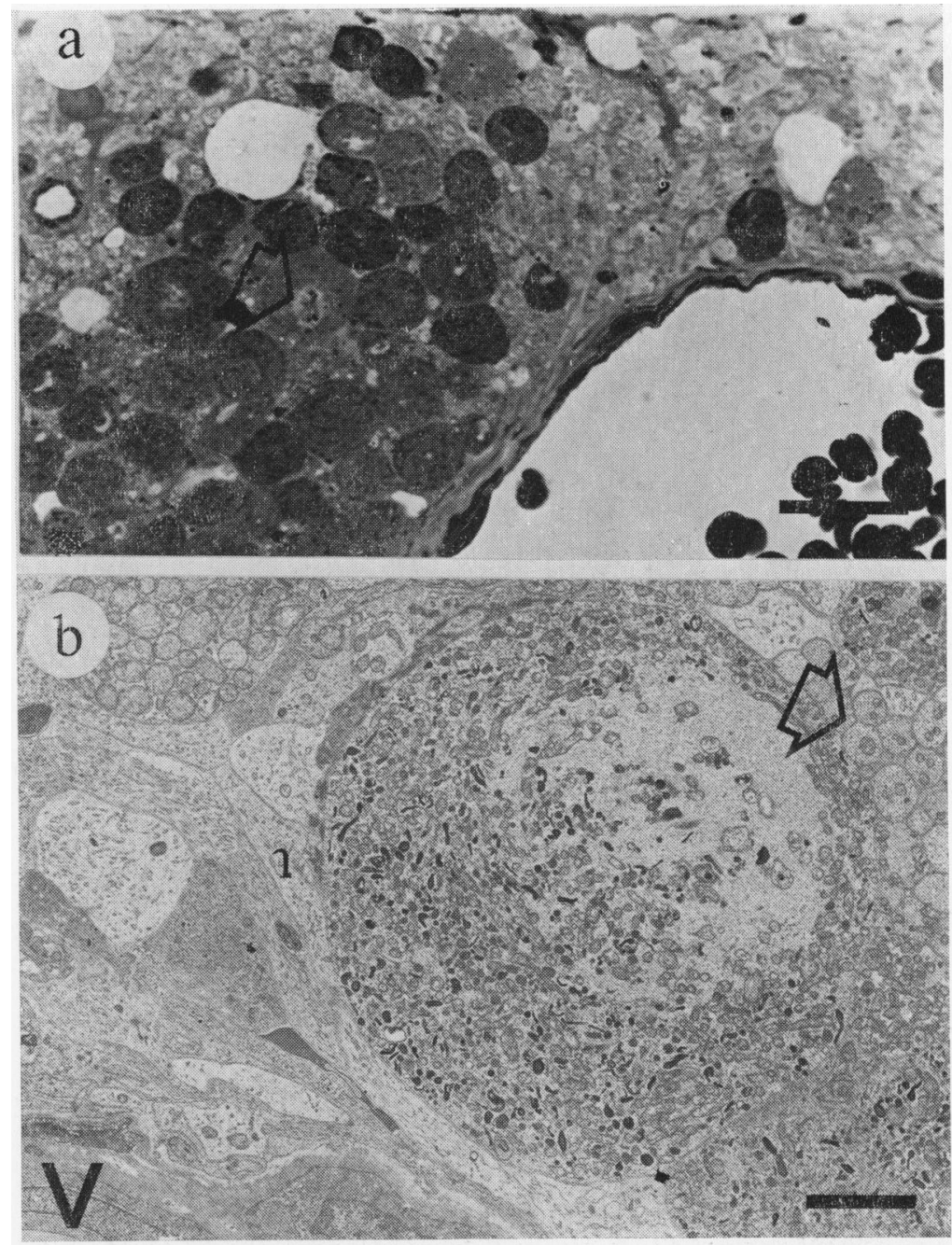

their lateral processes and become rounded and darkly pigmented (Fig. 8d). Macrophages were observed in the choroid associated with these damaged cells.

Three types of vascular lesions were observed, which were, in order of increasing severity, endothelial sloughing, endothelial loss with exudates, and small haemorrhages (Fig. 9). Endothelial sloughing was observed in vessels of all sizes but in large vessels was often highly focal and only in areas immediately adjacent to melanocytes (Fig. 9a). In smaller vessels, where endothelial cell loss and leakage of vessels contents had occurred small clots of platelets could be seen associated with the former areas of leakage (Fig. 9b). Exudates from many of these lesions had resulted in disorganisation of adjacent tissues.

\section{Discussion}

In any analysis of radiation induced damage to a biological system a fundamental requirement is the separation of acute radiation induced change in and adjacent to the absorption sites from the secondary tissue responses to such primary lesions. The present study involves the examination of damage produced by nonionising radiation 20 hours after exposure, and therefore much of the pattern of histological disturbance is due to tissue recriganisation in response to injury.

\section{RETINAL ABSORPTION SITES AND PRIMARY} DAMAGE

The retinal pigment epithelium is the major site of absorption of both argon and krypton radiation, 


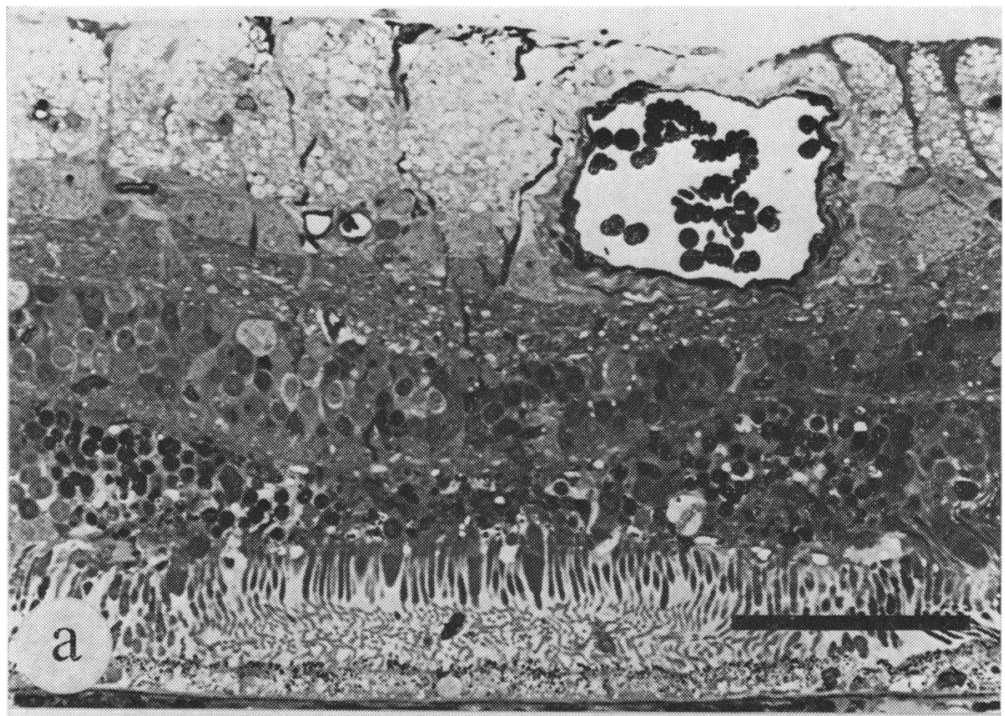

Fig. 7 (a) Light micrograph of $a$ vein 20 hours after irradiation by a krypton laser. In this lesion damage is confined to the outer

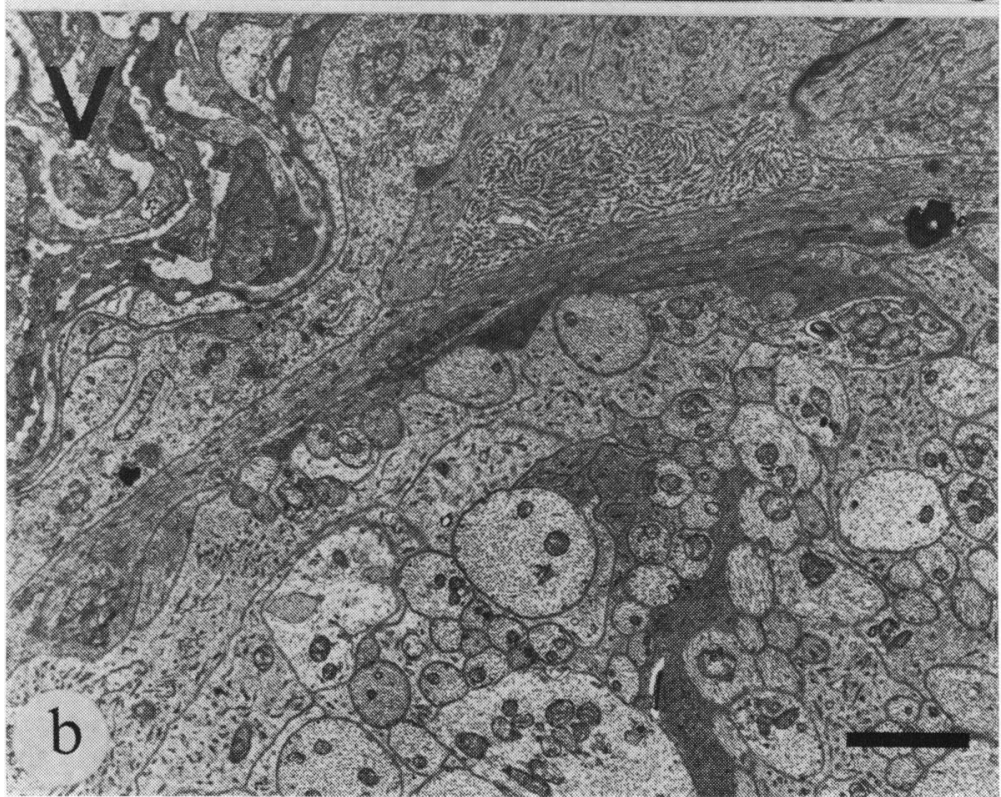
layers of the retina and no swollen axons are observed. The marker is $100 \mu \mathrm{m}$. (b) Electron micrograph of the axons adjacent to the irradiated vein (V) shown in (a). The marker is $2 \mu \mathrm{m}$

and in the time domain of clinical exposures all damage results from thermal processes. The most quoted studies of absorption of retinal pigment epithelium and choroid as a function of wavelengths show that, at the argon wavelength $(488 \mathrm{~nm}), 73 \%$ of the incident energy will be absorbed, while only $65 \%$ of absorption will occur at that of krypton $(641 \mathrm{~nm})$ (Geeraets and Berry, 1968). This 15\% differential in absorption between the 2 wavelengths seems inadequate to explain the factor of 2 between argon and krypton exposure energies for an equal retinal response in the macula and when exposing intraretinal vessels. However, our results indicate that in argon exposures very little energy passes through the pigment epithelium, so that in comparative studies it is the relative absorption of this layer alone that should be considered. Such measurements have been carried out by Gabel and colleagues (1977), who show that in human pigment epithelium there is a $40 \%$ differential in absorption between these 2 wavelengths and that at both wavelengths $33 \%$ less energy is absorbed in the perimacular 
Fig. 8 (a) Light micrograph of pigment epithelium and choroid in an unirradiated area showing the discontinuous array of choroidal melanocytes. (b) Light micrograph of a flat preparation of choroid showing the ameboid like processes of the choroidal melanocytes. The marker is $100 \mu \mathrm{m}$. (c) Light micrograph of unirradiated choroidal melanocytes showing their pigment distribution and the collagenous interstitial elements. (d) Light micrograph of krypton irradiated choroidal melanocytes showing abnormal cell profiles with pigment clumping, and the presence of both macrophages and oedema. The marker is $10 \mu \mathrm{m}$

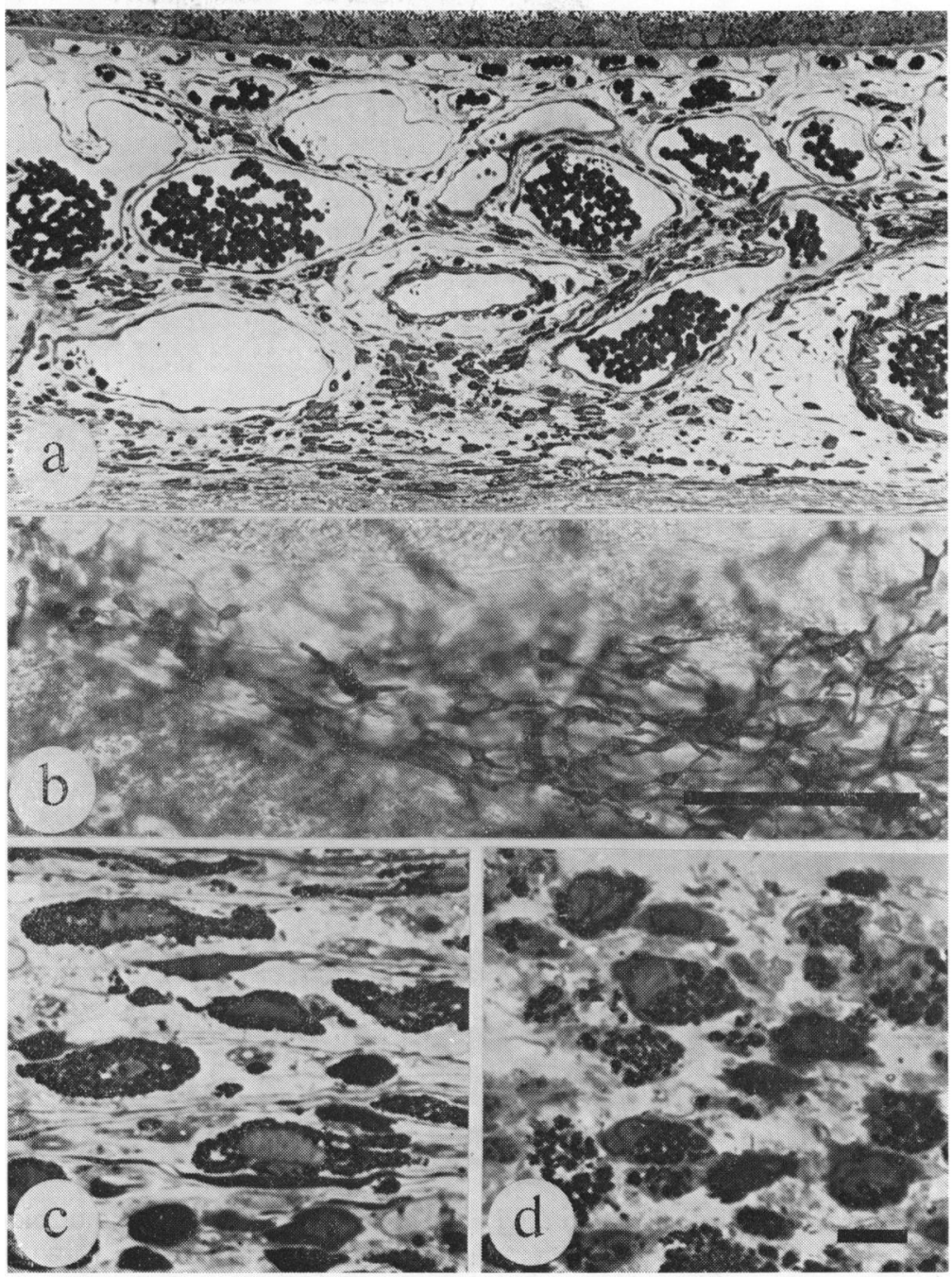

region than in the macula. Differences of this magnitude would be consistent with our observations. Further factors may have arisen due to differentials in energy distributions within the beams of the 2 systems used (Borland et al., 1978). Beam energy profiles were not determined, but observations indicated that neither of the beams had a Gaussian distribution of energy.

The absorption characteristics of the macular pigment have been determined as a function of wavelength and retinal location both in vivo (Ruddock, 1963) and in vitro (Gabel and Birngruber, 1979). While the absolute absorption varies between both individuals (Bone and Sparrock, 1971; Gabel and Birngruber, 1979) and races (Ishak, 1952), the relative absorption as a function of wavelength is remarkably constant. It has been shown that between $15 \%$ and $80 \%$ of the incident energy at the argon wavelength is absorbed in this pigment but less than $1 \%$ is absorbed at that of krypton. A similar differential in relative absorption exists when haemoglobin is irradiated, where, depending on the state of oxygenation, between 10 and 50 times more light is absorbed at $488 \mathrm{~nm}$ than at $630 \mathrm{~nm}$ (L'Esperance, 1975b).

In argon exposures these intraretinal absorption systems attenuate the radiation falling upon the pigment epithelium, and therefore damage in this layer is less marked in the fovea and under retinal vessels (Bowbyes et al., 1973). Very little krypton radiation is absorbed in either of these chromatophores, and only about $15 \%$ of the incident energy 


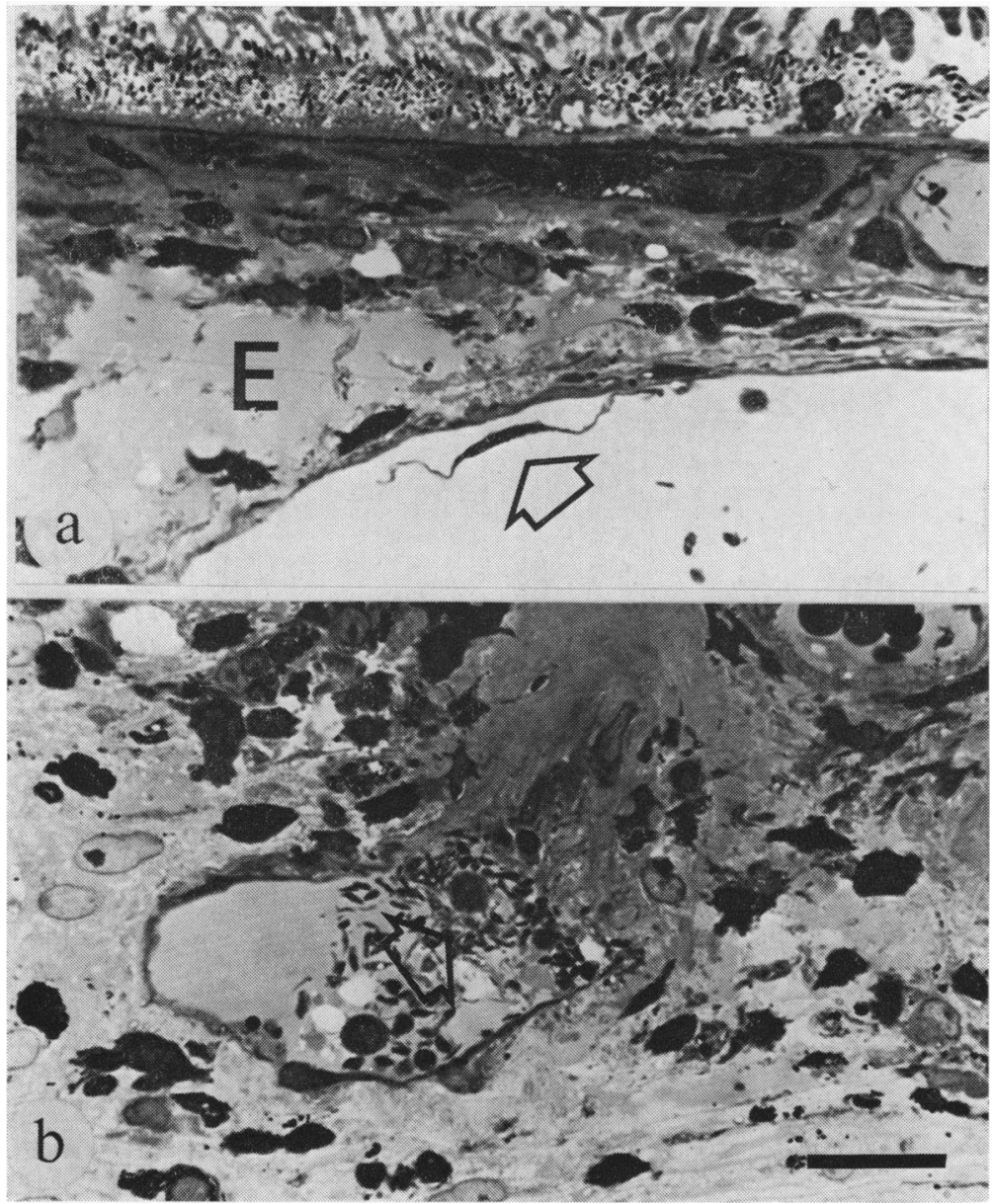

Fig. 9 (a) (b) Light micrographs of choroidal vessels 20 hours after irradiation with a krypton laser. (a) A small vascular lesion showing endothelial cell sloughing (arrowed) adjacent to an area of oedema and tissue disruption $(E)$. (b) $A$ more severe vascular lesion showing clotting (arrowed) subsequent to a choroidal haemorrhage, and the presence of both oedema and denatured melanocytes in the surrounding tissue. The marker is $20 \mu \mathrm{m}$

at this wavelength is dissipated within the pigment epithelium. It is therefore not surprising that in these exposures absorption within the choroidal melanocytes is of sufficient magnitude to induce damage to this layer (Figs. 8, 9).

The location of the various damage sites in the present study are clearly coincident with the sites of absorption. However, in determining the degree of damage associated with each site, not only is the relative absorption important but also the path length in which such absorption occurs. In general for any given value of absorption the shorter distance in which it occurs the greater the absorbed energy density and, above threshold, the greater the associated damage. If the thermal tolerance levels are similar for all cells throughout the retina, then damage associated with absorption by the pigment epithelium should be the most severe, as the pigment in this layer is confined to the apical $3 \mu \mathrm{m}$ of the epithelial cells, while in most cases both macular and blood pigments are more diffusely distributed. In the present study this concept is supported by the large regions of coagulated photoreceptor cells adhering to the pigment epithelium throughout both argon and krypton irradiated areas (Figs. 3, 4, 5, 7). The extensive disturbances in the inner retinal layers in argon exposures of the macula arise through bulk tissue displacement by secondary processes of fluid leakage. Secondary processes also amplify the damage site in argon irradiation of the intraretinal vessels.

The absorption parameters of the choroid are extremely difficult to describe, as the local variations are enormous (Fig. 8). Individual choroidal melanocytes are relatively large cells, being about $80 \mu \mathrm{m}$ in length and $10 \mu \mathrm{m}$ in depth, and can be extremely densely pigmented. This discontinuous array of highly absorbing cells probably accounts for the focal distribution of vascular lesions seen in the krypton irradiations, where up to $85 \%$ of the 
energy incident on the pigment epithelium passes through into the choroid. In the present study all choroidal changes associated with argon irradiations were confined to the choriocapillaris and resulted from thermal dissipation of energy absorbed within the pigment epithelium.

\section{SECONDARY RESPONSES TO LASER IRRADIATION}

The responses of retinal cells to various types of injury are a characteristic of the cells and not the injury. Thus the differences in topographic pathology between lesions induced by lasers of different wavelengths arise through either variations in the degree of damage to individual cell types or variations in the cell types damaged. In the present study within 20 hours of exposure in both argon and krypton lesions the pigment epithelial cells immediately adjacent to the irradiated area were undergoing changes in order to contain and clear the damaged site. The rounded cells protruding into the intra receptor matrix eventually bud off Bruch's membrane and join the blood-borne macrophages in clearing the debris of coagulated cells. These wandering epithelial cells are formed either by budding from multinucleate cells (Marshall and Mellerio, 1970) or by mitotic division (Ham et al., 1978), though in the present study we did not observe any mitotic figures.

The responses observed in irradiated photoreceptor cells were of degeneration and decay, and even in this short postexposure period many cells had lost large portions of their outer segments due to activity of macrophages. The degenerative changes resulting in the abnormally dense staining of the inner connecting fibres of some photoreceptor cells were shown to be extremely rapid in a previous study (Marshall et al., 1975). Both the angular inclinations with respect to the axis of radiation and the length of these degenerative fibres confirm that they result from secondary degenerative processes within the cell bodies of photoreceptor cells. Primary damage resulting from intrafibre absorption was observed in the innermost part of the fibre layer of Henle in the most central argon exposures, but this damage never resulted in the complementary production of densely stained fibres radiating to remote outer portions of receptor cells. In many of these inner retinal lesions the exudates arising from adjacent damaged cells resulted in a cleavage of inner and outer retinal layers along the line of the outer plexiform layer.

The axonal swellings seen in the nerve fibre layer in areas of argon irradiation of intraretinal vessels were the most striking secondary response in the inner retina (Figs. 5, 6). Terminal swellings on either side of interrupted axons in areas of intense photocoagulation have been previously reported (Okun and Collins, 1962; Marshall and Mellerio, 1967), but it was not appreciated until recently that such swellings arise through the interruption of the bidirectional flow of axoplasmic constituents along the axon. In an autoradiographic study of both laser and ischaemic lesions in pig retina it has been demonstrated that the swelling of damaged axons occurs as a passive response to the damming of axonal flow (McLeod et al., 1977). The observations in the present study are identical with those of McLeod and colleagues in that swelling due to interruption of retrograde transport in the isolated axonal segment adjacent to the optic disc was always larger than that due to orthograde flow in the segment attached to the cell body. The significance of this type of axonal damage to field loss following irradiation would be very difficult to assess, as in all lesions in this study some normal looking axons were present in the exposed areas.

The presence of oedema and macrophages in the choroid in areas of krypton irradiation is clearly a secondary response to changes in the vascular elements in this tissue, and represents an early phase of wound repair (Fig. 9).

Laser technology is now capable of producing reliable systems with a variety of emission wavelengths, and therefore there is a potential for the selective exploitation of ocular absorption sites. To do this effectively a better understanding of the particular aspects of photocoagulation which result in the beneficial changes in various types of neovascular lesions is essential. At present none of the therapeutic procedures are fully understood in terms of their reaction sequence. Thus in the successful treatment of disciform lesions we do not know whether the therapeutic response is $1^{\circ}$ or $2^{\circ}$ in either the new vessels, the pigment epithelium, the choroid, or some combination of these, or which tissue absorption is important in generating this change. Even more complex conceptual problems arise in optimising peripheral ablation treatment. Several authors (see L'Esperance, 1975c) have suggested that the large areas of retinal destruction result in a reduced metabolic demand and that this in turn influences neovascular atrophy. However, given the dual circulating system of the retina with a choroidal supply to the photoreceptor cells, and an intraretinal system which feeds all the inner retinal layers, it is difficult to understand how destruction of the photoreceptor cells and the underlying choroid would achieve this end in respect of the intraretinal vascular system. A further consideration is the increase in bulk fluid flow through the retinal pigment epithelium following 
photocoagulation, which would result in loss from the vitreous of any diffusible agent which may be influencing blood vessel behaviour (Foulds, 1976).

With this uncertainty it is impossible to predict the relative clinical values of lasers with different wavelengths with their different absorption characteristics, and different spectra of tissue damage, but as we now have the capability to damage selected regions of the retina it is hoped that future clinical studies will optimise the selection of laser wavelengths for specific retinal diseases. In particular the krypton laser allows damage to be restricted to the outer retina and choroid.

We thank Miss E. Clarke and Mr P. L. Ansell for technical assistance and Miss Julie Bramley for secretarial help. We would like to express our gratitude to Lasertek for the loan of their krypton laser. We are also indebted to the British Committee for the Prevention of Blindness, the Garrick Trust, and the Wellcome Trust for gifts of capital equipment.

\section{References}

Bird, A. C. (1974). Recent advances in the treatment of senile disciform macular degeneration by photocoagulation. British Journal of Ophthalmology, 58, 367-376.

Bone, R. A., and Sparrock, J. M. B. (1971). Comparison of macular pigment densities in human eyes. Vision Research, 11, 1057-1064.

Borland, R. G., Brennan, D. H., Marshall, J., and Viveash, J. P. (1978). The role of fluorescein angiography in the detection of laser-induced damage to the retina : a threshold study for Q-switched, neodymium and ruby lasers. Experimental Eye Research, 27, 471-493.

Bowbyes, J. A., Hamilton, A. M., Bird, A. C., Blach, R. K., Marshall, J., and Kohner, E. M. (1973). The argon laser: The effect on retinal tissues and its clinical applications. Transactions of the Ophthalmological Societies of the United Kingdom, 93, 439-453.

Foulds, W. S. (1976). Clinical significance of trans-scleral fluid transfer. Transactions of the Ophthalmological Societies of the United Kingdom, 96, 290-308.

Gabel, V. P., and Birngruber, R. (1979). Klinishe folgerungen aus der xanthophylleinlargerung in der Netzhautmitte. In press.

Gabel, V. P., Birngruber, R., and Hillenkamp, F. (1977). Individuelle Unterschiede der Lichtabsorption am Augenhintergrund in sichtbaren und infraroten Spectralbereich, Deutsche Ophthalmologische Gessellschaft, Berichtuberdie 74. Zusammenkunft, 418-421.

Gass, J. D. M. (1971). Photocoagulation of macular lesions. Transactions of the American Academy of Ophthalmology and Otolaryngology, 75, 580-608.

Gass, J. D. M. (1973). Drusen and disciform macular detachment and degeneration. Archives of Ophthalmology, 90, 206-217.

Geeraets, W. J., and Berry, B. S. (1968). Ocular spectral characteristics as related to hazards from lasers and other light sources. American Journal of Ophthalmology, 66, 15-20.

Ham, W. T., Ruffolo, J. J., Mueller, H. A., Clarke, A. M., and Moon, M. E. (1978). Histologic analysis of photochemical lesions produced in rhesus retina by short wavelength light. Investigative Ophthalmology and Visual Science, 17, 1029-1035.

Ishak, I. G. H. (1952). The photopic luminosity curve for a group of fifteen Egyptian trichromats. Journal of the Optical Society of America, 42, 529-534.

L'Esperance, F. A. (1968). An ophthalmic argon laser photocoagulation system, design, construction, and laboratory investigations. Transactions of the American Ophthalmological Societies, 66, 827-904.

L'Esperance, F. A. (1975a). Ocular Photocoagulation: $a$ Stereoscopic Atlas, pp. 146-153. C. V. Mosby: Saint Louis.

L'Esperance, F. A. (1975b). Ocular Photocoagulation: $a$ Stereoscopic Atlas, p. 29. C. V. Mosby: Saint Louis.

L'Esperance, F. A. (1975c). Ocular Photocoagulation: $a$ Stereoscopic Atlas, p. 152. C. V. Mosby: Saint Louis.

Little, H. L. (1973). Argon laser therapy of diabetic retinopathy. Documenta Ophthalmologica (Den Haag) Proc. Series, 1, 87-95, in François, J. (Ed.), Symposium on light-coagulation Argon laser and Xenon, Arc. Ghent, 15-16 June 1972.

Little, H. L., Zweng, H. C., and Peabody, R. R. (1970). Argon laser slit lamp retinal photocoagulation. Transactions of the American Academy of Ophthalmology and Otolaryngology, 74, 85-97.

McLeod, D., Marshall, J., Kohner, E. M., and Bird, A. C. (1977). The role of axoplasmic transport in the pathogenesis of retinal cotton-wool spots. British Journal of Ophthalmology, 61, 177-191.

Manson, N., Marshall, J., Mellerio, J., and Smart, D. (1972). Comments on histological studies of gas laser lesions in humans and possible non-linear optical phenomena, together with experiments using a tuneable dye laser. Modern Problems in Ophthalmology, 10, 114-128.

Marshall, J. (1970). Thermal and mechanical mechanisms in laser damage to the retina. Investigative Ophthalmology, 9, 2, 97-115.

Marshall, J., Hamilton, A. M., and Bird, A. C. (1974). Intra retinal absorption of argon laser irradiation in human and monkey. Experientia (Basel), 26, 1335-1337.

Marshall, J., Hamilton, A. M., and Bird, A. C. (1975). Histopathology of ruby and argon laser lesions in monkey and human retina: a comparative study. British Journal of Ophthalmology, 59, 610-630.

Marshall, J., and Mellerio, J. (1967). Pathological development of retinal laser photocoagulations. Experimental Eye Research, 6, 303-308.

Marshall, J., and Mellerio, J. (1970). Laser irradiation of retinal tissue. British Medical Bulletin, 26, 156-160.

Okun, E., and Collins, E. M. (1962). Histopathology of experimental photocoagulation in the dog eye: II Production of cytoid body lesions. American Journal of Ophthalmology, 54, 786.

Ruddock, K. H. (1963). Evidence for macular pigmentation from colour matching data, Vision Research, 3, 417-429. 